\title{
Cryptogenic fibrosing alveolitis and lung cancer: the BTS study
}

\author{
J M Harris, ${ }^{1}$ I D A Johnston, ${ }^{2}$ R Rudd, ${ }^{3}$ A J Newman Taylor, ${ }^{1}$ P Cullinan'
}

\begin{abstract}
${ }^{1}$ Occupational and Environmental Medicine, National Heart and Lung Institute, Imperial College, London, UK: ${ }^{2}$ Nottingham University Hospitals, Queens Medical Centre Campus, Nottingham, UK; ${ }^{3}$ London Lung Cancer Group, London, UK
\end{abstract}

Correspondence to: Dr J Harris, Occupational and Environmental Medicine,

National Heart \& Lung Institute, Imperial College, 1B Manresa Road, London SW3 6LR, UK; jessica.harris@imperial.ac.uk

Received 19 June 2009 Accepted 6 November 2009 Published Online First 29 November 2009

\begin{abstract}
Background: The risk of lung cancer is often reported to be increased for patients with cryptogenic fibrosing alveolitis (CFA).

Methods: Vital status was sought for all 588 members of the British Thoracic Society (BTS) cryptogenic fibrosing alveolitis (CFA) study 11 years after entry to the cohort. Observed deaths due to lung cancer were compared with expected deaths using age-, sex- and period-adjusted national rates. The roles of reported asbestos exposure and smoking were also investigated.
\end{abstract}

Results: 488 cohort members (83\%) had died; 46 (9\%) were certified to lung cancer (ICD9 162). The standardised mortality ratio (SMR) was $7.4(95 \% \mathrm{Cl} 5.4$ to 9.9$)$. Stratified analysis showed increased lung cancer mortality among younger subjects, men and ever smokers. Using an independent expert panel, 25 cohort members (4\%) were considered to have at least moderate exposure to asbestos; the risk of lung cancer was increased for these subjects (SMR 13.1 (95\% Cl 3.6 to 33.6$)$ ) vs $7.2(95 \% \mathrm{Cl}$ 5.2 to 9.7$)$ for those with less or no asbestos exposure). Ever smoking was reported by $448(73 \%)$ of the cohort and was considerably higher in men than in women $(92 \%$ vs $49 \% ; p<0.001)$. Most persons who died from lung cancer were male (87\%), and all but two (96\%) had ever smoked. Ever smokers presented at a younger age (mean 67 vs 70 years; $p<0.001$ ) and with less breathlessness (12\% smokers reported no breathlessness vs $5 \%$ never smokers; $p=0.02$ ).

Conclusions: These findings confirm an association between CFA and lung cancer although this relationship may not be causal. The high rate of smoking and evidence that smokers present for medical attention earlier than non-smokers suggest that smoking could be confounding this association.

Increased lung cancer mortality among patients with cryptogenic fibrosing alveolitis (CFA) or idiopathic pulmonary fibrosis (IPF) has been reported in numerous studies, with prevalences as high as $48.2 \%{ }^{1}{ }^{1}$ Fourteen reports of lung cancer mortality in series of patients with this disease are shown in table 1, with a pooled estimate of $17.3 \%$ (95\% CI $11.2 \%$ to $25.9 \%$ ). Dependent on the era of publication, these studies include a variable mixture of patients with CFA and IPF, although there is no clear evidence that this has had any bearing on the reported associations with lung cancer.

In contrast, population-based co-mortality studies from the USA ${ }^{2}$ and $\mathrm{UK}^{3}$ have not confirmed an increased risk of lung cancer. In the US study, Wells et $a l^{2}$ reported a lower rate of lung cancer mortality among people who died from pulmonary fibrosis (proportional mortality $4.8 \%$ ) than among those who died from chronic obstructive pulmonary disease $(10.1 \%)$ or asbestosis $(26.6 \%)$. Similar findings were reported from the UK study; ${ }^{3}$ the proportions of certificates which also mentioned lung cancer were consistently lower for certificates with mention of postinflammatory pulmonary fibrosis (3\%), other alveolar and parietoalveolar pneumopathy (6\%), silicosis $(7 \%)$ or coal workers' pneumoconiosis (8\%) than those with a mention of asbestosis (43\%).

The aim of this study was to investigate the relationship between CFA and lung cancer in more detail by consideration of death certificate details for an established UK-wide cohort of patients with CFA.

\section{METHODS \\ BTS study of CFA}

On 1 December 1990, recruitment to the British Thoracic Society (BTS) CFA study began. ${ }^{45}$ The study concerns patients with the clinical syndrome of CFA as then diagnosed rather than patients exclusively with IPF or usual interstitial pneumonitis (UIP) according to current diagnostic criteria devised since the study started.

All respiratory physicians in England, Scotland and Wales were invited to enter prospectively all new patients with CFA over the following 2 years. CFA was defined either on histological grounds or according to clinical criteria. These criteria included any evidence of bilateral interstitial chest radiographic shadowing with bilateral basal inspiratory crackles and lung function parameters compatible with CFA (ie, a restrictive and/or gas transfer effect). Open lung biopsy specimens were taken in $12 \%$ of the study population and transbronchial biopsies in $28 \%$; $60 \%$ had no histological examination. ${ }^{45}$

A total of 415 respiratory physicians were contacted and $330(79.5 \%)$ agreed to participate in the study. After the 2-year enrolment period, 588 patients with a definite diagnosis of CFA identified by one of 150 chest physicians were initially followed up until November 1996 or until death, if earlier.

\section{Vital status}

A specific code was introduced for CFA/IPF with the 9th revision of the International Classification of Diseases in 1979 (ICD9): 516.3 Idiopathic Fibrosing Alveolitis; in version 10 the code is: J84.1 Other Interstitial Pulmonary Disease with Fibrosis. The vital status at 31 December 2001 was requested from the Office for National Statistics (ONS) for all 588 cohort members, together with all listed causes of death coded to ICD9. By this date, $488(83.4 \%)$ had died, $97(16.7 \%)$ were not known to have died and ONS could not trace three cohort members. 
Table 1 Lung cancer mortality among patients with cryptogenic fibrosing alveolitis (CFA)/idiopathic pulmonary fibrosis (IPF)

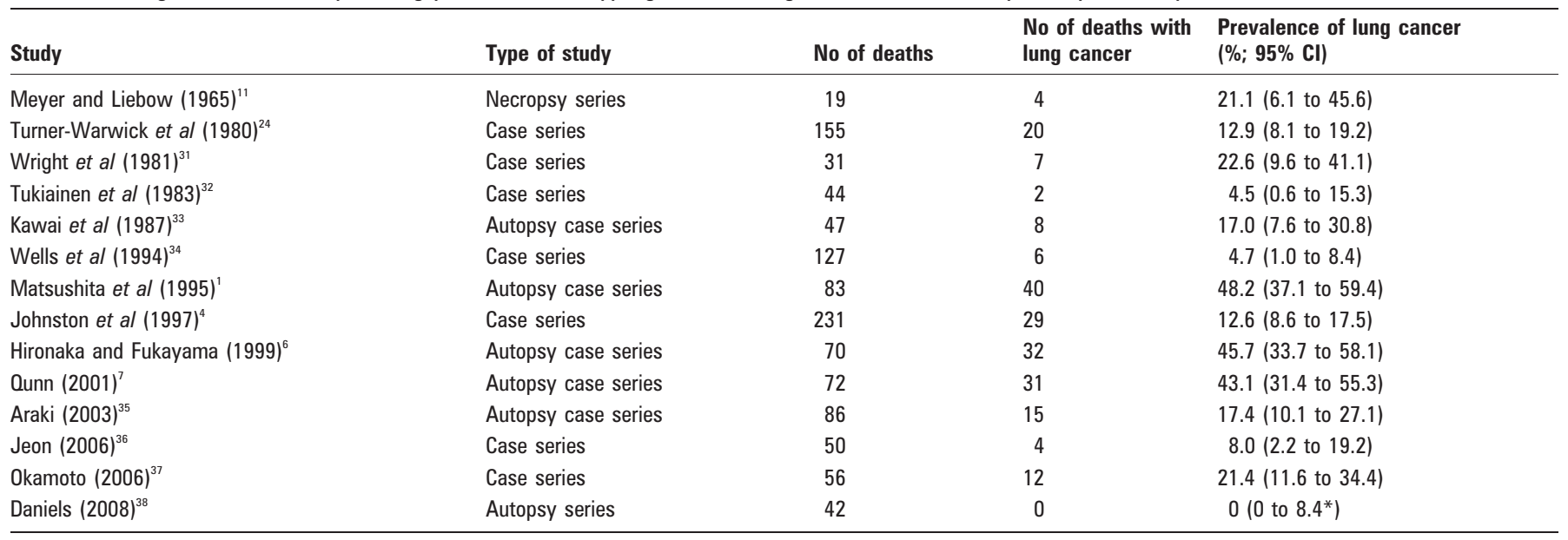

${ }^{*}$ One-sided $97.5 \%$ confidence interval.

\section{Asbestos exposure}

Information on reported exposure to asbestos and to other organic and inorganic dusts was collected by questionnaire administered at enrolment. Details on duration, era and intensity of exposure, along with the specific job, industry or hobby involved, were recorded. The BTS CFA study eligibility criteria excluded any individual who, in the opinion of the participating chest physician, had occupational exposures which would be accepted as a sufficient basis for a diagnosis of pneumoconiosis for the purposes of state compensation. ${ }^{4}$ However, a number of the occupational histories identified possible exposure to these dusts, especially asbestos exposure $(\mathrm{n}=87 ; 15.0 \%)$. In the present study, six consultant respiratory physicians were independently asked to grade each subject as having had no, mild, moderate or heavy exposure to asbestos based on the reported job/industry together with era and duration of exposure. Any cohort member who was considered by at least four physicians to have had "moderate" exposure was classified as such $(n=25)$. The risk of mortality from lung cancer was calculated separately for these categories and, in each case, compared with those with less or no reported exposure to asbestos. A stratified analysis of increased duration of reported asbestos exposure ( $>10$ years) was also conducted.

\section{Smoking}

Information on smoking was also collected in the BTS questionnaire. Subjects were defined as current, ex-smokers or non-smokers.

\section{Statistical methods}

$\chi^{2}$ tests, $t$ tests, Mann-Whitney tests and $\chi^{2}$ tests for trend were used to compare people who died from lung cancer with all other deaths. Comparisons between the observed deaths due to lung cancer (ICD9 162) and the expected number of deaths were made. Expected deaths were calculated by the application of age- and sex-specific rates for the years 1990-2001 for England, Wales and Scotland. The results were then expressed as standardised mortality ratios (SMR; observed/expected deaths) with exact $95 \%$ confidence intervals calculated using the Poisson distribution. Five subjects were excluded from the cohort analysis as the vital status was unknown $(n=3)$ or the recorded date of exit from the cohort was before the entry date $(n=2)$. Analyses were stratified by severity at presentation measured by exercise grade (normal exercise grade, breathless up hill and normal pace on level or breathless at normal pace on level versus breathless when walking 100 yards slowly or breathless at rest), age, sex, smoking history and reported asbestos exposure. In order to attempt to examine the increased probability of lung cancer being diagnosed among subjects with CFA - for example, through increased investigations - and also to investigate the latency of these diseases, univariate and stratified results were repeated after excluding individuals who died within 1 year of entry to the cohort and then those who died within 2, 3, 4 or 5 years of entry to the cohort. Analysis was conducted using SAS (SAS Institute Inc, Cary, North Carolina, USA) and Stata (Stata Corporation, Texas, USA) software.

\section{Risk of other cancer}

In order to assess the specificity of any association with lung cancer, the methodology was repeated for all cancer deaths excluding lung cancer (ie, ICD9 codes 140-239 excluding 162, and ICD10 codes C00-D48 excluding C34).

\section{RESULTS}

\section{Vital status}

By 31 December 2001, 488 (83.4\%) had died, 333 (68.4\%) with idiopathic fibrosing alveolitis (ICD9 516.3) listed as a mentioned cause and 46 (9.4\%) with lung cancer (ICD9 162). Twenty-three of those who died had both idiopathic fibrosing alveolitis and lung cancer mentioned on the death certificates, $6.9 \%$ of all deaths which mentioned idiopathic fibrosing alveolitis.

The median survival was 2.56 years (95\% CI 2.17 to 3.06 ). This was significantly less for those who died from lung cancer (2.05 years (95\% CI 1.39 to 3.18$)$ ) than for the remaining members of the cohort (2.61 years (95\% CI 2.20 to 3.13 ); $p=0.01)$. Thirteen of the 46 deaths from lung cancer $(28.3 \%)$ occurred within 1 year of presentation, 21 (47.8\%) within 2 years and $29(63.0 \%)$ within 3 years.

Those who died from lung cancer were more likely to be male and were younger than those who died from other causes (table 2). Less breathlessness and cough was reported by people who died from lung cancer and the proportion of smokers was significantly higher. A higher proportion of people who died from lung cancer reported exposure to asbestos $(23.9 \%$ vs $14.6 \% ; p=0.09)$ and other inorganic dusts (43.5\% vs $27.1 \%$; $\mathrm{p}=0.02)$ 
Table 2 Lung cancer mortality and all other causes of mortality in the BTS cryptogenic fibrosing alveolitis cohort

\begin{tabular}{|c|c|c|c|}
\hline & Recorded cause of deat & & \\
\hline & $\begin{array}{l}\text { Lung cancer (ICD9 162) } \\
(\mathrm{n}=46)\end{array}$ & $\begin{array}{l}\text { All other causes } \\
(n=442)\end{array}$ & p Value \\
\hline Sex, n (\%) male & $40(87.0 \%)$ & $281(63.6 \%)$ & $<0.001$ \\
\hline Mean (SD) age at entry (years) & $65.8(7.7)$ & $69.6(9.3)$ & 0.01 \\
\hline Mean (SD) age at death (years) & $68.4(7.0)$ & $72.2(9.2)$ & 0.01 \\
\hline Presentation & & & \\
\hline Breathlessness, n (\%) & $37(80.4 \%)$ & $403(91.2 \%)$ & 0.02 \\
\hline $\begin{array}{l}\text { Median (range) duration of breathlessness } \\
\text { (months) }\end{array}$ & $12(1-60)$ & $10(1-84)$ & 0.85 \\
\hline Cough, n (\%) & $28(60.9 \%)$ & $337(76.2 \%)$ & 0.02 \\
\hline Median (range) duration of cough (months) & $6(1-60)$ & $7(1-84)$ & 0.93 \\
\hline Chronic bronchitis $\left(\mathrm{MRC}^{*}\right), \mathrm{n}(\%)$ & $10(21.7 \%)$ & $86(19.5 \%)$ & 0.72 \\
\hline Exercise grade, $\mathrm{n}(\%)$ & $8(17.4 \%)$ & $36(8.1 \%)$ & $0.12(0.01 \dagger)$ \\
\hline Normal & $17(37.0 \%)$ & $129(29.2 \%)$ & \\
\hline Breathless up hill & $8(17.4 \%)$ & $95(21.5 \%)$ & \\
\hline Breathless on level & $11(23.9 \%)$ & $137(31.0 \%)$ & \\
\hline Breathless walking slowly & $2(4.4 \%)$ & $45(10.2 \%)$ & \\
\hline Breathless at rest & & & \\
\hline Clubbing, $\mathrm{n}(\%)$ & $31(67.4 \%)$ & $219(49.7 \%)$ & 0.02 \\
\hline Ischaemic heart disease, $\mathrm{n}(\%)$ & $14(31.3 \%)$ & $77(17.5 \%)$ & 0.03 \\
\hline Exposures & & & \\
\hline Smoking history, $\mathrm{n}(\%)$ & & & $<0.001$ \\
\hline Current & $19(41.3 \%)$ & $72(16.3 \%)$ & $(<0.001 \dagger)$ \\
\hline Ex-smoker & $25(54.4 \%)$ & $260(58.8 \%)$ & \\
\hline Non-smoker & $2(4.4 \%)$ & $110(24.9 \%)$ & \\
\hline Ever smoked, $\mathrm{n}(\%)$ & $44(95.7 \%)$ & $332(75.1 \%)$ & 0.002 \\
\hline
\end{tabular}

*Defined as "a cough productive of sputum for at least three months of two consecutive years". ${ }^{39}$

$\dagger p$ value for trend.

There was also some evidence that those who died from lung cancer were more likely to present with other conditions; 37 (80.4\%) of the 46 lung cancer deaths reported at least one of the specific conditions detailed within the patient's general medical history compared with $287 / 442(64.9 \%)$ of those who died from all other causes $(p=0.03)$.

Compared with national statistics and after taking age, sex and period into account, there were 7.40 times more deaths from lung cancer observed than expected (95\% CI 5.42 to 9.88). This excess reduced to 1.79 (95\% CI 0.72 to 3.69) once those who died within 5 years of presentation were excluded (fig 1).

\section{Stratified analyses}

Stratified analyses showed increased lung cancer mortality among younger subjects (aged $<68.75$ years), men, current and ex-smokers (fig 2) and those with reported asbestos exposure (table 3). Few differences were observed when the data were stratified by severity on presentation. When individuals who died within specific periods of presentation were excluded, estimates of the risk of lung cancer mortality tended towards unity and in most cases were no longer significantly raised once subjects who died within 5 years of presentation were omitted (data not shown). Only among the 122 younger subjects and the 44 current smokers who were still alive 5 years after presentation were there significantly more deaths from lung cancer than expected (6 observed, 1.90 expected, SMR $3.16(95 \%$ CI 1.16 to 6.87); 5 observed, 0.82 expected, SMR 6.08 (95\% CI 1.97 to 14.18$)$, respectively)

\section{Asbestos exposure}

The analyses stratified by asbestos exposure showed an increased risk of death from lung cancer among the group with the higher exposure, with ratios of observed to expected lung cancer deaths 2-3 times higher in the exposed subjects. However, in most analyses, estimates tended to be wide with confidence intervals that overlapped (table 3). Again, estimates tended towards the null when those who died soon after presentation were excluded (data not shown).

\section{Smoking}

A large majority of the cohort members were current or exsmokers $(76.2 \%$, table 4$)$. This proportion was similar for those who had died by 31 December 2001 (77.0\%) and those who were not known to have died by that date $(73.5 \%, p=0.41)$. Of 46 lung cancer deaths, only two $(4.3 \%)$ were never smokers and only six (13.0\%) were women. Median survival was longer among current smokers ( 3.81 years (95\% CI 2.75 to 4.80$)$ ) than among non-smokers (2.34 years (95\% CI 1.37 to 3.54$)$ ) or exsmokers $(2.24$ years (95\% CI 1.84 to 2.95$)) ; p=0.26$.

Reported rates of smoking were higher among men $(91.7 \%)$ than women $(49.3 \% ; \mathrm{p}<0.001)$. Men were significantly more likely to have died than women $(86.5 \%$ vs $78.0 \%$; $p=0.01)$. Deceased men were significantly more likely to be defined as current or ex-smokers on presentation $(91.9 \%$ vs $48.5 \%$; $\mathrm{p}<0.001)$ and to have lung cancer recorded on their death certificates $(12.5 \%$ vs $3.6 \% ; p=0.001)$. Smoking rates were significantly higher for those who had died from lung cancer than those who died free from lung cancer $(95.7 \%$ vs $75.1 \%$; $p=0.002$ ).

Smokers presented at a younger age than non-smokers (mean 67.0 years (95\% CI 66.1 to 67.8 ) vs 70.6 years (95\% CI 68.7 to 72.5); $\mathrm{p}<0.001)$. There was also evidence that current or past smokers presented earlier, since less breathlessness was reported by smokers on presentation (54 (12.1\%) ever smokers reported 
Figure 1 Standardised mortality ratios and $95 \%$ confidence intervals for lung cancer mortality within complete and restricted datasets. Results are based on the whole dataset $(n=583)$, together with restricted datasets after excluding ( $n=412,328,270,227,186$, respectively). those who died within specific periods

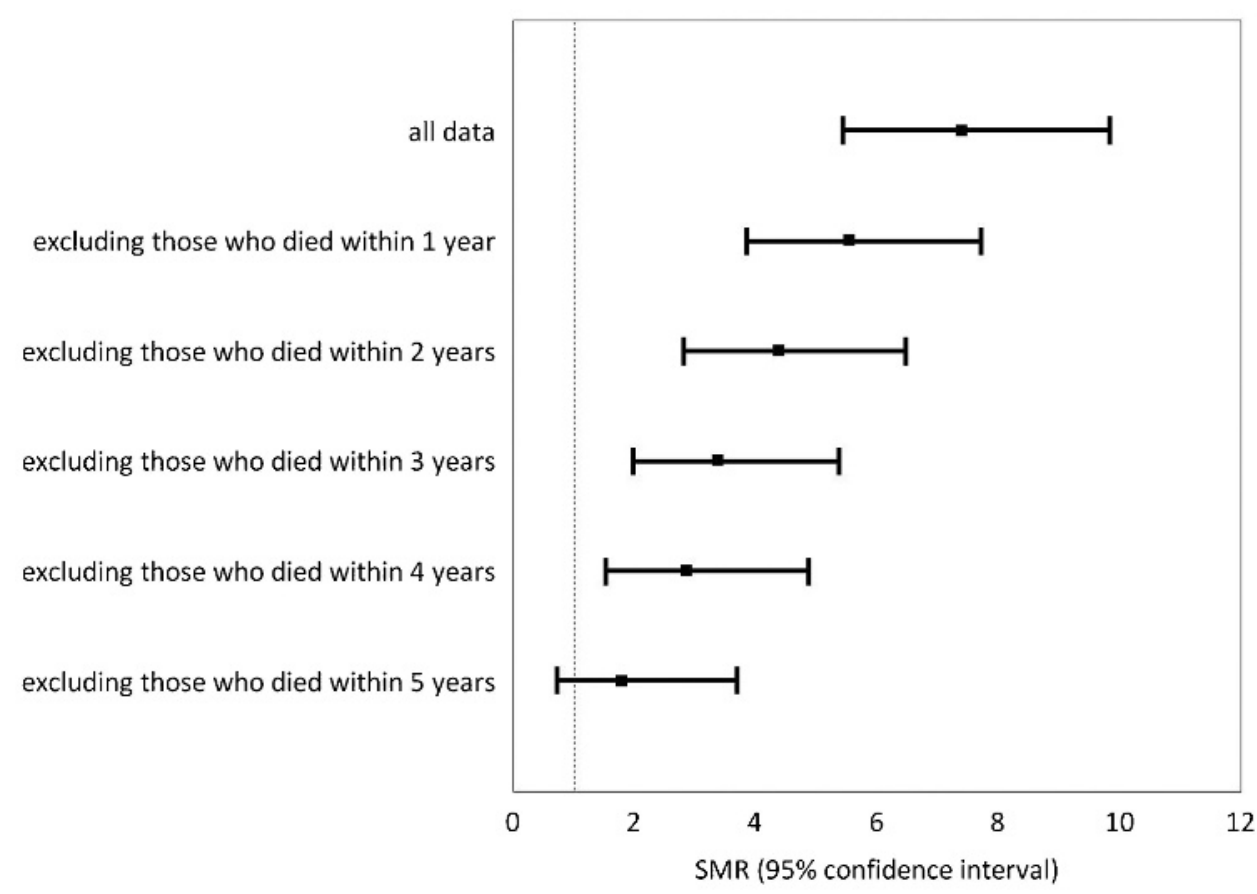

no breathless vs $7(5.0 \%)$ never smokers; $p=0.02)$. Ratios of observed to expected deaths for smokers stratified by exercise grade on presentation did not follow a clear trend (table 5).

\section{Risk of other cancer}

There were 24 observed deaths due to other cancers: carcinomatosis $(n=8)$, stomach $(n=3)$, oesophagus $(n=2)$, colon $(n=2)$, rectum $(n=2)$, pancreas $(n=2)$, liver, prostate, breast, cervix, ovaries (all $n=1$ ) and 17.07 expected deaths. The SMR for all cancers excluding lung cancer was 1.41 (95\% CI 0.90 to 2.09). When lung cancers were included (total 70 deaths), the SMR for all cancers was 3.01 (95\% CI 2.34 to 3.80 ).

\section{DISCUSSION}

The mortality analysis of the members of the BTS CFA cohort identified that there were 46 deaths from lung cancer among 488 people who died (9.4\%) and 23 (6.9\%) deaths with both idiopathic fibrosing alveolitis and lung cancer mentioned on the death certificates, a figure only marginally higher than the UK co-mortality estimate (5.6\%).

It is plausible that the population-based co-mortality studies $^{23}$ were limited by incomplete listings of pulmonary fibrosis on the death certificates, and diagnostic misclassification may have also contributed to the lack of association between IPF and lung cancer. A far higher prevalence of lung cancer was reported
Figure 2 Standardised mortality ratios and $95 \%$ confidence intervals for lung cancer mortality within complete and restricted datasets stratified by smoking. Black lines (with squares) represent nonsmokers, dark grey lines (with diamonds) represent ex-smokers and light grey lines (with squares) represent current smokers. The results are based on the whole dataset ( $n=136$ non-smokers, 337 exsmokers and 110 current smokers), together with restricted datasets after excluding those who died within specific periods ( $\mathrm{n}=88,72,62,54,40$ nonsmokers; 236, 182, 147, 118, 102 exsmokers; 88, 75, 61, 55, 44 current smokers).

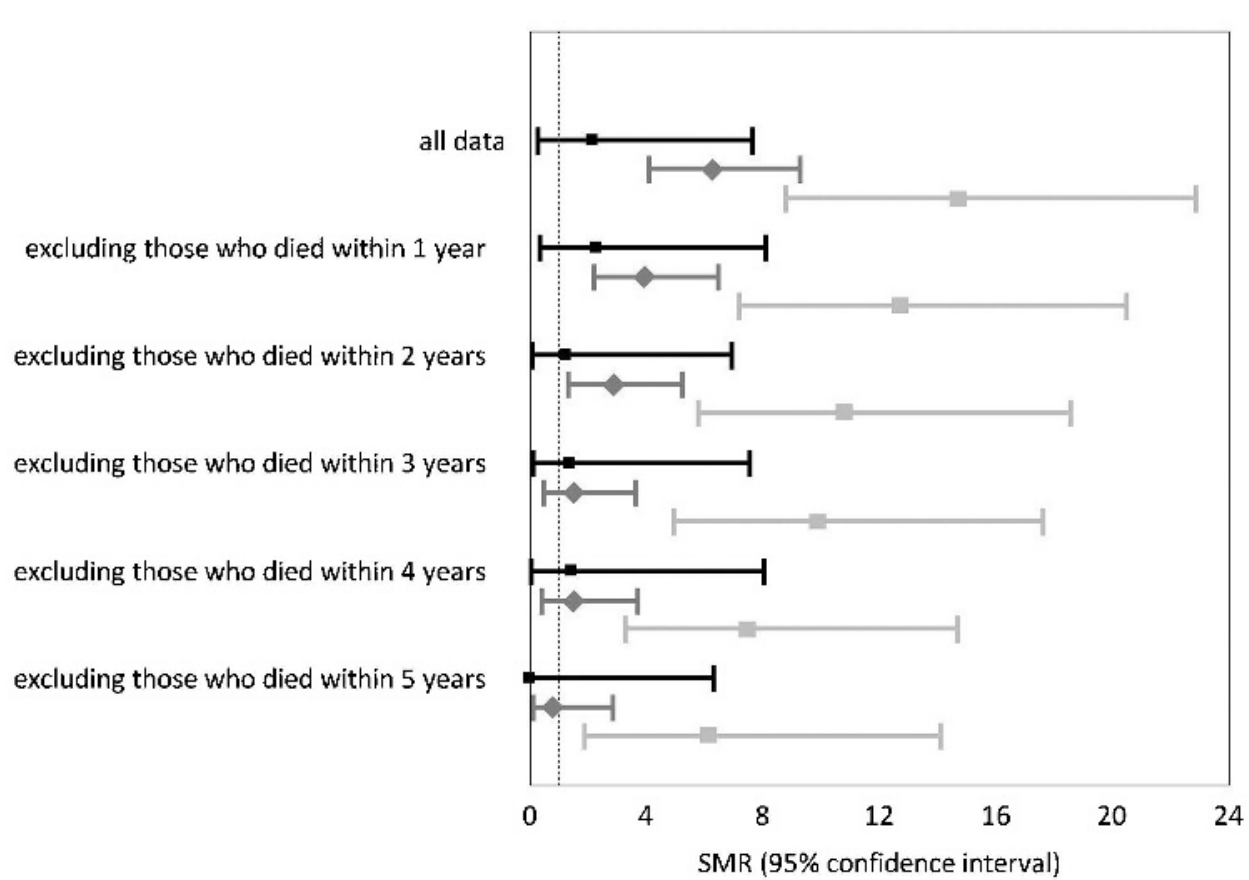


Table 3 Observed and expected deaths from lung cancer for members of the BTS cryptogenic fibrosing alveolitis cohort

\begin{tabular}{|c|c|c|c|c|c|}
\hline & $\mathbf{N}$ & $\begin{array}{l}\text { Person-years } \\
\text { at risk }\end{array}$ & $\begin{array}{l}\text { Observed } \\
\text { deaths }\end{array}$ & $\begin{array}{l}\text { Expected } \\
\text { deaths }\end{array}$ & SMR (95\% CI) \\
\hline All & 583 & 2296.30 & 46 & 6.21 & 7.40 (5.42 to 9.88$)$ \\
\hline Younger (<68.75 years) & $293(50.3 \%)$ & 1423.91 & 28 & 2.61 & $10.72(7.10$ to 15.45$)$ \\
\hline Older $(\geqslant 68.75$ years $)$ & 290 & 872.40 & 18 & 3.58 & 5.02 (2.98 to 7.95$)$ \\
\hline Men & $370(63.5 \%)$ & 1369.62 & 40 & 4.93 & $8.12(5.80$ to 11.05$)$ \\
\hline Women & 213 & 926.68 & 6 & 1.28 & $4.67(1.72$ to 10.20$)$ \\
\hline Non-smokers & $136(23.3 \%)$ & 516.82 & 2 & 0.94 & $2.13(0.26$ to 7.69$)$ \\
\hline Ex-smokers & $337(57.8 \%)$ & 1270.76 & 25 & 3.98 & 6.28 (4.06 to 9.27$)$ \\
\hline Current smokers & $110(18.9 \%)$ & 508.72 & 19 & 1.29 & 14.67 (8.83 to 22.92 ) \\
\hline $\begin{array}{l}\text { Less severe symptoms on } \\
\text { presentation* }\end{array}$ & $375(64.4 \%)$ & 1786.28 & 33 & 4.75 & $6.95(4.78$ to 6.96$)$ \\
\hline $\begin{array}{l}\text { More severe symptoms on } \\
\text { presentation } \dagger\end{array}$ & 207 & 499.47 & 13 & 1.46 & 8.93 (4.74 to 15.23$)$ \\
\hline No reported asbestos exposure & $494(85.0 \%)$ & 1967.87 & 35 & 5.18 & $6.76(4.71$ to 9.40$)$ \\
\hline Reported asbestos exposure & 87 & 327.86 & 11 & 1.03 & $10.65(5.32$ to 19.06$)$ \\
\hline Less than "moderate" exposure & $557(95.7 \%)$ & 2193.17 & 42 & 5.88 & $7.15(5.15$ to 9.66$)$ \\
\hline "Moderate" exposure or more & 25 & 98.38 & 4 & 0.30 & 13.12 (3.57 to 33.59$)$ \\
\hline Less than 10 years exposure & $543(93.5 \%)$ & 2168.52 & 38 & 5.78 & 6.57 (4.65 to 9.02$)$ \\
\hline At least 10 years exposure & 38 & 127.21 & 8 & 0.43 & 18.58 (8.03 to 36.66$)$ \\
\hline
\end{tabular}

in other studies. The highest were those by Matsushita et $a l,{ }^{1}$ Hironaka and Fukayama ${ }^{6}$ and Qunn, ${ }^{7}$ with a lung cancer prevalence of $48.2 \%, 45.7 \%$ and $43.1 \%$, respectively. All of these studies were autopsy studies conducted in Japan and none was blinded. These estimates may be elevated by the increased probability of the presence of lung cancer being detected once an autopsy has taken place. The aim in this present study was to avoid these potential limitations by investigating lung cancer mortality within a large cohort of patients with CFA/IPF.

Careful comparison of observed deaths with the number expected based on age-, sex- and period-specific national lung cancer mortality rates identified that the lung cancer deaths within this CFA cohort were significantly more frequent than expected, with an SMR of 7.40 (95\% CI 5.42 to 9.88).

There was also an excess of deaths due to other cancers (24 deaths), although this was not statistically significant (SMR 1.41 (95\% CI 0.90 to 2.09$)$ ). When respiratory cancers were included, the SMR for all cancers was 3.01 (95\% CI 2.34 to 3.80). This was higher than the estimate obtained by Le Jeune et $a l^{8}$ who reported a rate ratio of 1.51 (95\% CI 1.20 to 1.90$)$ among 1064 cases of IPF compared with matched controls.

Stratified lung cancer mortality analysis identified an increased risk among younger subjects, men, smokers (particularly current smokers) and those who reported asbestos exposure. These findings largely agree with a study by Aubry et $a l^{9}$ who compared a group of 24 patients with IPF and lung cancer with 63 patients with IPF only and observed that patients with IPF and lung cancer were more likely to be men and smokers. Other studies have also demonstrated considerable excess of lung cancer among male patients with CFA/IPF, ${ }^{16}$ 10-13 far greater than the male:female ratio observed in CFA/IPF.

In a large study of CFA cases and matched controls extracted from general practice data reported by Hubbard et al, ${ }^{14}$ the rate ratio of lung cancer among CFA cases was 7.31 (95\% CI 4.47 to 11.93), similar to the SMR for the BTS CFA cohort. The observed increase remained when the analysis was restricted to current smokers only (RR 7.36 (95\% CI 1.54 to 35.19)). ${ }^{14}$ When only current smokers were considered for the BTS CFA cohort, the SMR was much higher (14.67 (95\% CI 8.83 to 22.92$)$ ) as no adjustments for smoking habits among the population mortality data were possible.

The simple and most widely held explanation for the excess of lung cancer observed in the BTS cohort is that CFA causes lung cancer. However, other explanations need to be considered. First, non-small cell lung cancers probably start to grow from the first malignant cell approximately 10 years before clinical manifestation while, for small cell cancers, the interval is approximately 3 years, ${ }^{15}$ suggesting that many of the patients who subsequently died from lung cancer would have had the disease much further back in time and almost certainly at the time of presentation. Thirteen (28.3\%) of the lung cancer deaths occurred within a year of presentation, 21 (47.8\%) within 2 years and $29(63.0 \%)$ within 3 years. All stratified analyses were segregated by survival time, and the resulting estimates of lung cancer risk tended towards the null when those who died soon after presentation were excluded from each analysis. If CFA does cause lung cancer, it might reasonably be expected that these estimates of risk would increase with survival time

Table 4 Smoking rates across subgroups of the BTS cryptogenic fibrosing alveolitis cohort

\begin{tabular}{lccc}
\hline & All $(\mathbf{n}=\mathbf{5 8 8})$ & Women $(\mathbf{n = 2 1 5})$ & Men (n= 373) \\
\hline Alive on 31 December 2001 & $71 / 97(73.2 \%)$ & $25 / 47(53.2 \%)$ & $46 / 50(92.0 \%)$ \\
Died by 31 December 2001 & $376 / 488(77.1 \%)$ & $81 / 167(48.5 \%)$ & $295 / 321(91.9 \%)$ \\
Died from lung cancer & $44 / 46(95.7 \%)$ & $5 / 6(83.3 \%)$ & $39 / 40(97.5 \%)$ \\
Died free of lung cancer & $332 / 442(75.1 \%)$ & $76 / 161(47.2 \%)$ & $256 / 281(91.1 \%)$ \\
All & $448 / 588(76.2 \%)$ & $106 / 215(49.3 \%)$ & $342 / 373(91.7 \%)$ \\
\hline
\end{tabular}


Table 5 Standardised mortality ratios stratified by severity of disease (ever smokers)

\begin{tabular}{|c|c|c|c|c|}
\hline & \multicolumn{4}{|c|}{ Smokers $(n=447)$} \\
\hline & No & $\begin{array}{l}\text { Observed } \\
\text { deaths }\end{array}$ & $\begin{array}{l}\text { Expected } \\
\text { deaths }\end{array}$ & SMR (95\% CI) \\
\hline Normal & 54 & 8 & 1.01 & 7.91 (3.42 to 15.61$)$ \\
\hline Breathless up hill & 159 & 17 & 1.95 & 8.73 (5.08 to 13.96$)$ \\
\hline Breathless on level & 98 & 8 & 1.16 & 6.90 (2.98 to 13.59$)$ \\
\hline Breathless walking slowly & 106 & 9 & 1.00 & 9.01 (4.12 to 17.08 ) \\
\hline Breathless at rest & 30 & 2 & 0.16 & $12.69(1.51$ to 45.15$)$ \\
\hline
\end{tabular}

SMR, standardised mortality ratio.

since those patients who survive longer theoretically experience an increased time in which to develop lung cancer. It is also likely that the first fibroblastic focus may occur many years before diagnosis, suggesting that it is possible that the tumour and fibrosis occur concurrently.

Second, all but two of the lung cancer deaths (95.7\%) occurred among smokers and the prevalence of past or current smoking was high in this cohort $(76.2 \%)$. A similar prevalence of smoking was reported by Hubbard et al ${ }^{16}(77 \%)$, Baumgartner et $a l^{17}(72 \%)$ and Antoniou et $a l^{18}(75 \%)$, although other studies have reported much lower rates (29-37\%). ${ }^{814} 19$ Two of these three studies were based on information extracted from a large general practice database and the third study was based on data extracted from an Oxygen Registry. Smoking data may be incomplete in these studies or, in the case of the last, inaccurate since patients may have underestimated their smoking in order to get access to oxygen. Alternatively, these variations may reflect subtle differences in the use of terms such as CFA and IPF. Many studies have identified smoking as a risk factor for CFA/IPF with a population attributable risk of $49.1 \% .{ }^{20}$

There was also some evidence from this cohort that ever smokers present earlier, based on less reported breathlessness. Smokers were also younger at presentation. It is possible that smokers are more likely to undergo radiography, which may lead to CFA being systematically and preferentially diagnosed among subjects who smoke or who have ever smoked, introducing ascertainment bias. There was some evidence that survival among current smokers was longer than in never or past smokers, an observation reported earlier by King et $a l^{21}$ who reported significantly higher survival for current smokers and by Antoniou et $a^{18}$ who observed lower mortality for current smokers than for former smokers. In this latter paper the investigators examined survival after adjustment for severity and concluded that this improved outcome suggested that current smokers present with less severe CFA. Smoking may play two distinct roles in pulmonary fibrosis-increasing the patient's probability of presenting for medical attention and directly increasing the risk of patients with CFA to develop lung cancer.

The third possibility is that the observed excess of lung cancer deaths is due in part to misclassification of asbestosis; the associations between asbestosis and lung cancer are well established. ${ }^{22}{ }^{23}$ Recorded occupational data highlight that some patients $(14.9 \%)$ reported exposure to asbestos. Lung cancer mortality was 2-4 times higher for those with asbestos exposure.

The final consideration - which arises in part from the observation of increased smoking among the patients with CFA and from the observation of fewer symptoms on presentation among past or current smokers-may explain the excess lung cancer mortality observed within this and other CFA/IPF series, including notable studies by Turner-Warwick et $a l^{24}$ and Hubbard et al. ${ }^{14}$ This increased risk of lung cancer may be driven by an inherent ascertainment bias (an example of Berkson's bias ${ }^{25}$ ). Berkson recognised that hospitalised patients are not representative of the general population and that people with two or more conditions are more likely to attend hospital than those with only one condition, with the potential for producing false associations. Within the BTS CFA cohort it is plausible that the increased observation of lung cancer may have arisen by increased ascertainment among smokers, or among those already with lung cancer. Certainly the high rates of smoking, especially among the men who accounted for the majority of lung cancer deaths, would support this hypothesis.

The associations between lung cancer and other fibrotic diseases are more established; many studies have reported increased lung cancer risk among patients with asbestosis. ${ }^{22} 2326$ An increased risk of lung cancer has also been reported among patients with silicosis ${ }^{28} 29$ and scleroderma. ${ }^{30}$ These studies of asbestosis and silicosis are based upon population data, arguably providing an improved setting in which to examine the pathogenesis of these fibrotic diseases. Most studies of CFA/IPF which have reported increased risks of lung cancer have findings based on hospital case series where inherent ascertainment bias is more probable.

In summary, evidence from this and other studies suggests an association between CFA and lung cancer. However, in the light of the questionable temporality of this association, the confounding effects of smoking, the high rate of asbestos exposure among this cohort and the possibility of inherent ascertainment bias highlighted by Berkson, ${ }^{25}$ the causal relationship of CFA and lung cancer remains questionable.

Acknowledgements: We are grateful to the 150 members of the British Thoracic Society who participated in this study supported by the Morriston Davies Trust and the consultant physicians who assisted with the asbestos grading exercise: Professor Sherwood Burge (Birmingham Heartlands Hospital), Dr Adrian Draper (St George's Hospital, London), Dr Clive McGavin (Plymouth Nuffield Hospital) and Professor Athol Wells (Royal Brompton Hospital) along with ANT and PC (authors). We also thank Robin Prescott and J C Chalmers (University of Edinburgh), Professor Corbett McDonald and Mrs R Ryan and Mrs V E Johnston.

Funding: $\mathrm{JH}$ is supported by the Colt Foundation.

Competing interests: None.

Provenance and peer review: Not commissioned; externally peer reviewed.

\section{REFERENCES}

1. Matsushita H, Tanaka S, Saiki Y, et al. Lung cancer associated with usual interstitial pneumonia. Pathol Int 1995;45:925-32.

2. Wells C, Mannino DM. Pulmonary fibrosis and lung cancer in the United States: analysis of the multiple cause of death mortality data, 1979 through 1991. South Med J 1996;89:505-10.

3. Harris JM, Cullinan P, McDonald JC. Does cryptogenic fibrosing alveolitis carry an increased risk of death from lung cancer? J Epidemiol Community Health 1998;52:602-3

4. Johnston ID, Prescott RJ, Chalmers JC, et al. British Thoracic Society study of cryptogenic fibrosing alveolitis: current presentation and initial management. Fibrosing Alveolitis Subcommittee of the Research Committee of the British Thoracic Society. Thorax 1997:52:38-44. 
5. Rudd RM, Prescott RJ, Chalmers JC, et al. British Thoracic Society study on cryptogenic fibrosing alveolitis: response to treatment and survival. Thorax 2007;62:62-6.

6. Hironaka M, Fukayama M. Pulmonary fibrosis and lung carcinoma: a comparative study of metaplastic epithelia in honeycombed areas of usual interstitial pneumonia with or without lung carcinoma. Pathol Int 1999;49:1060-6.

7. Ounn L, Takemura T, Ikushima $\mathrm{S}$, et al. Hyperplastic epithelial foci in honeycomb lesions in idiopathic pulmonary fibrosis. Virchows Arch 2002;441:271-8.

8. Le Jeune I, Gribbin J, West J, et al. The incidence of cancer in patients with idiopathic pulmonary fibrosis and sarcoidosis in the UK. Respir Med 2007:101:2534-40.

9. Aubry MC, Myers JL, Douglas WW, et al. Primary pulmonary carcinoma in patients with idiopathic pulmonary fibrosis. Mayo Clin Proc 2002;77:763-70.

10. Park J, Kim DS, Shim TS, et al. Lung cancer in patients with idiopathic pulmonary fibrosis. Eur Respir J 2001;17:1216-9.

11. Meyer EC, Liebow AA. Relationship of interstitial pneumonia honeycombing and atypical epithelial proliferation to cancer of the lung. Cancer 1965;18:322-51.

12. Panos RJ, Mortenson RL, Niccoli SA, et al. Clinical deterioration in patients with idiopathic pulmonary fibrosis: causes and assessment. Am J Med 1990:88:396-404.

13. Nagai A, Chiyotani A, Nakadate $T$, et al. Lung cancer in patients with idiopathic pulmonary fibrosis. Tohoku J Exp Med 1992;167:231-7.

14. Hubbard R, Venn A, Lewis S, et al. Lung cancer and cryptogenic fibrosing alveolitis. A population-based cohort study. Am J Respir Crit Care Med 2000;161:5-8.

15. Geddes DM. The natural history of lung cancer: a review based on rates of tumour growth. Br J Dis Chest 1979;73:1-17.

16. Hubbard R, Lewis S, Richards K, et al. Occupational exposure to metal or wood dust and aetiology of cryptogenic fibrosing alveolitis. Lancet 1996;347:284-9.

17. Baumgartner KB, Samet JM, Stidley CA, et al. Cigarette smoking: a risk factor for idiopathic pulmonary fibrosis. Am J Respir Crit Care Med 1997;155:242-8.

18. Antoniou KM, Hansell DM, Rubens MB, et al. Idiopathic pulmonary fibrosis: outcome in relation to smoking status. Am J Respir Crit Care Med 2008;177:190-4.

19. Gustafson T, Dahlman-Hoglund A, Nilsson K, et al. Occupational exposure and severe pulmonary fibrosis. Respir Med 2007;101:2207-12.

20. Taskar VS, Coultas DB. Is idiopathic pulmonary fibrosis an environmental disease? Proc Am Thorac Soc 2006:3:293-8.

21. King TE Jr, Tooze JA, Schwarz MI, et al. Predicting survival in idiopathic pulmonary fibrosis: scoring system and survival model. Am J Respir Crit Care Med 2001;164:1171-81.

22. Mossman BT, Gee JB. Asbestos-related diseases. N Engl J Med 1989;320:1721-30.
23. Kishimoto $\mathbf{T}$, Okada K. The relationship between lung cancer and asbestos exposure. Chest 1988;94:486-90.

24. Turner-Warwick M, Lebowitz M, Burrows B, et al. Cryptogenic fibrosing alveolitis and lung cancer. Thorax 1980;35:496-9.

25. Berkson J. Limitations of the application of fourfold tables to hospital data. Biometrics Bull 1946;2:47-53.

26. Ontario Ministry of the Attorney General 0C1. Report of the Royal Commission on Matters of Health and Safety Arising From the Use of Asbestos in Ontario. 1984.

27. Saracci R. Asbestos and lung cancer: an analysis of the epidemiological evidence on the asbestos-smoking interaction. Int J Cancer 1977;20:323-31.

28. McDonald JC. Silica, silicosis, and lung cancer: an epidemiological update. App/ Occup Environ Hyg 1995;10:1056-63.

29. Weill H, McDonald JC. Exposure to crystalline silica and risk of lung cancer: the epidemiological evidence. Thorax 1996;51:97-102.

30. Hill CL, Nguyen AM, Roder D, et al. Risk of cancer in patients with scleroderma: a population based cohort study. Ann Rheum Dis 2003;62:728-31.

31. Wright PH, Heard BE, Steel SJ, et al. Cryptogenic fibrosing alveolitis: assessment by graded trephine lung biopsy histology compared with clinical, radiographic, and physiological features. $\mathrm{Br} J$ Dis Chest 1981;75:61-70.

32. Tukiainen $\mathbf{P}$, Taskinen $\mathrm{E}$, Holsti $\mathrm{P}$, et al. Prognosis of cryptogenic fibrosing alveolitis. Thorax 1983;38:349-55

33. Kawai T, Yakumaru K, Suzuki M, et al. Diffuse interstitial pulmonary fibrosis and lung cancer. Acta Pathol Jpn 1987;37:11-9.

34. Wells AU, Cullinan P, Hansell DM, et al. Fibrosing alveolitis associated with systemic sclerosis has a better prognosis than lone cryptogenic fibrosing alveolitis. Am J Respir Crit Care Med 1994;149:1583-90.

35. Araki T, Katsura H, Sawabe M, et al. A clinical study of idiopathic pulmonary fibrosis based on autopsy studies in elderly patients. Intern Med 2003;42:483-9.

36. Jeon K, Chung MP, Lee KS, et al. Prognostic factors and causes of death in Korean patients with idiopathic pulmonary fibrosis. Respir Med 2006;100:451-7.

37. Okamoto $\mathbf{T}$, Ichiyas $\mathrm{H}$, Ichikado $\mathrm{K}$, et al. Clinical analysis of the acute exacerbation in patients with idiopathic pulmonary fibrosis. J Japanese Respir Soc 2006;44:359-67.

38. Daniels CE, Yi ES, Ryu JH. Autopsy findings in 42 consecutive patients with idiopathic pulmonary fibrosis. Eur Respir J 2008;32:170-4.

39. Anon. Definition and classification of chronic bronchitis for clinical and epidemiological purposes. A report to the Medical Research Council by their Committee on the Aetiology of Chronic Bronchitis. Lancet 1965;1:775-9. 